\title{
E-Referral System to Improve Triaging and Referrals to an ENT Specialist Department
}

\author{
Yousuf Hussain, Antonia Tse, Surya Narayan
}

\section{East Lancashire Hospitals NHS Trust}

East Lancashire Hospitals W/HS NHS Trust

\section{BACKGROUND}

- It can be difficult to triage referrals from other departments unless specific ENT-relevant clinical information is provided.

\section{INTRODUCTION}

- Royal Blackburn Hospital houses a busy ENT department which caters for both elective and emergency cases.

- Urgent referrals are sent through to on call doctors within this department for triaging.

- Many of these referrals are verbally discussed with the first on call doctor, most of which originate from primary care, the emergency department or urgent care.

- Beyond this, there are referrals sent from inpatient wards which are triaged by the middle grade second on call doctor.

- Due to the nature of handwritten paper referrals, some critical information may be missing which makes it difficult to triage the requirement for the patients appropriately.

\section{AIM}

- To assess the quality of the referrals received with the current system (handwritten paper referrals) using the standardised hospital proforma.

- To consider an E-referral system already implemented by other departments in the same trust.

\section{METHODS}

- All faxed paper referrals were collected over a representative two week period and analysed for the inclusion of the information as shown below in Figure 1.

- Duplicates were excluded.

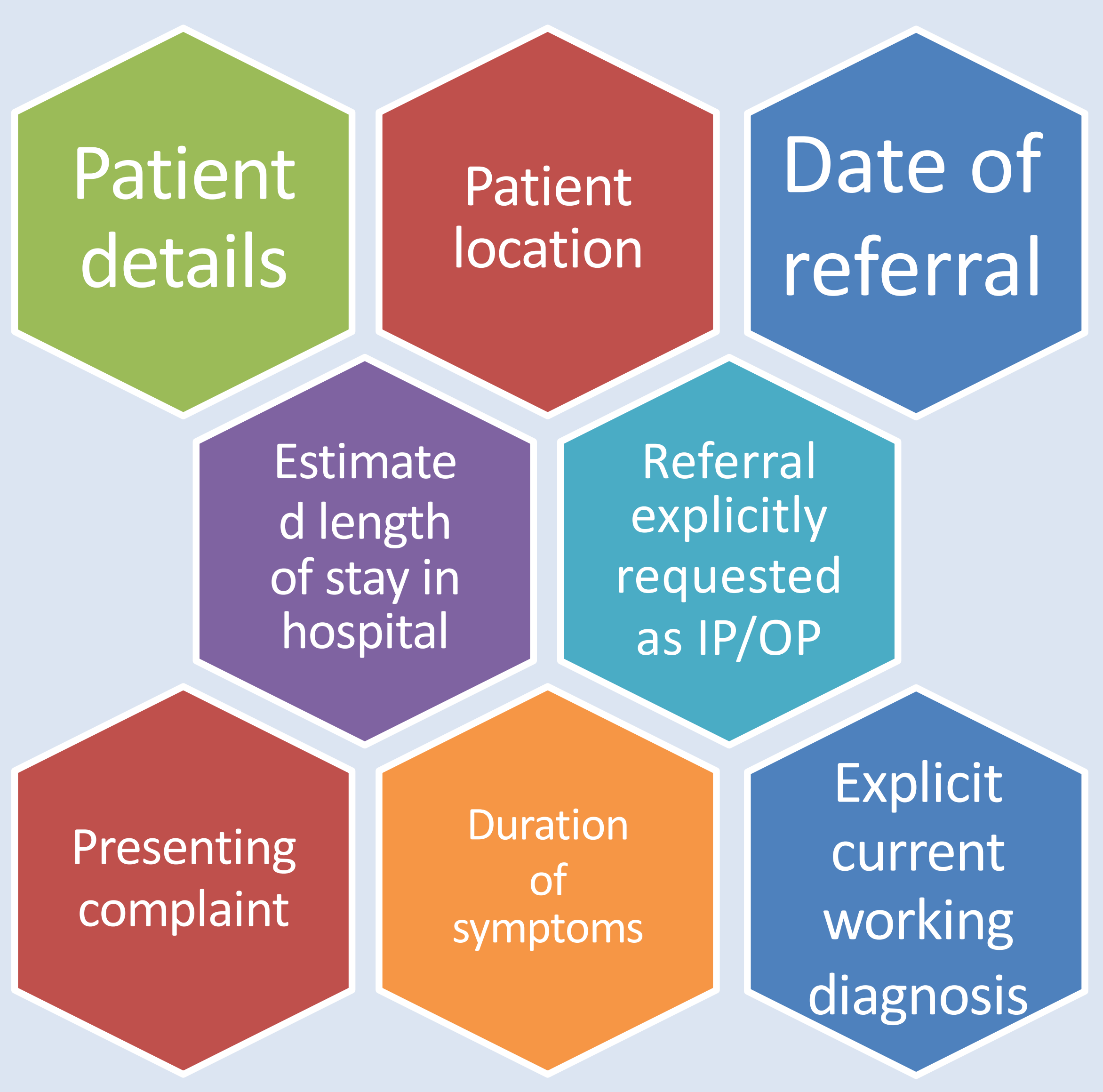

Figure 1. Data collected for inclusion for audit

\section{RESULTS}

- All paper referrals from wards and primary care faxed to secretaries over a 2 week period from 18/12/17 29/12/17.

- 19 referrals were found to meet the inclusion criteria, and after 5 duplicates were excluded, the remaining 14 referrals were analysed for the relevant information, displayed in figure 2 .

- There was good compliance of: patient details/referral date (100\%); presenting complaint (93\%); location (82\%) and working diagnosis (71\%).

- There was poor compliance of symptom duration (50\%), in/out-patient review (57\%) and expected length of stay (0\%).
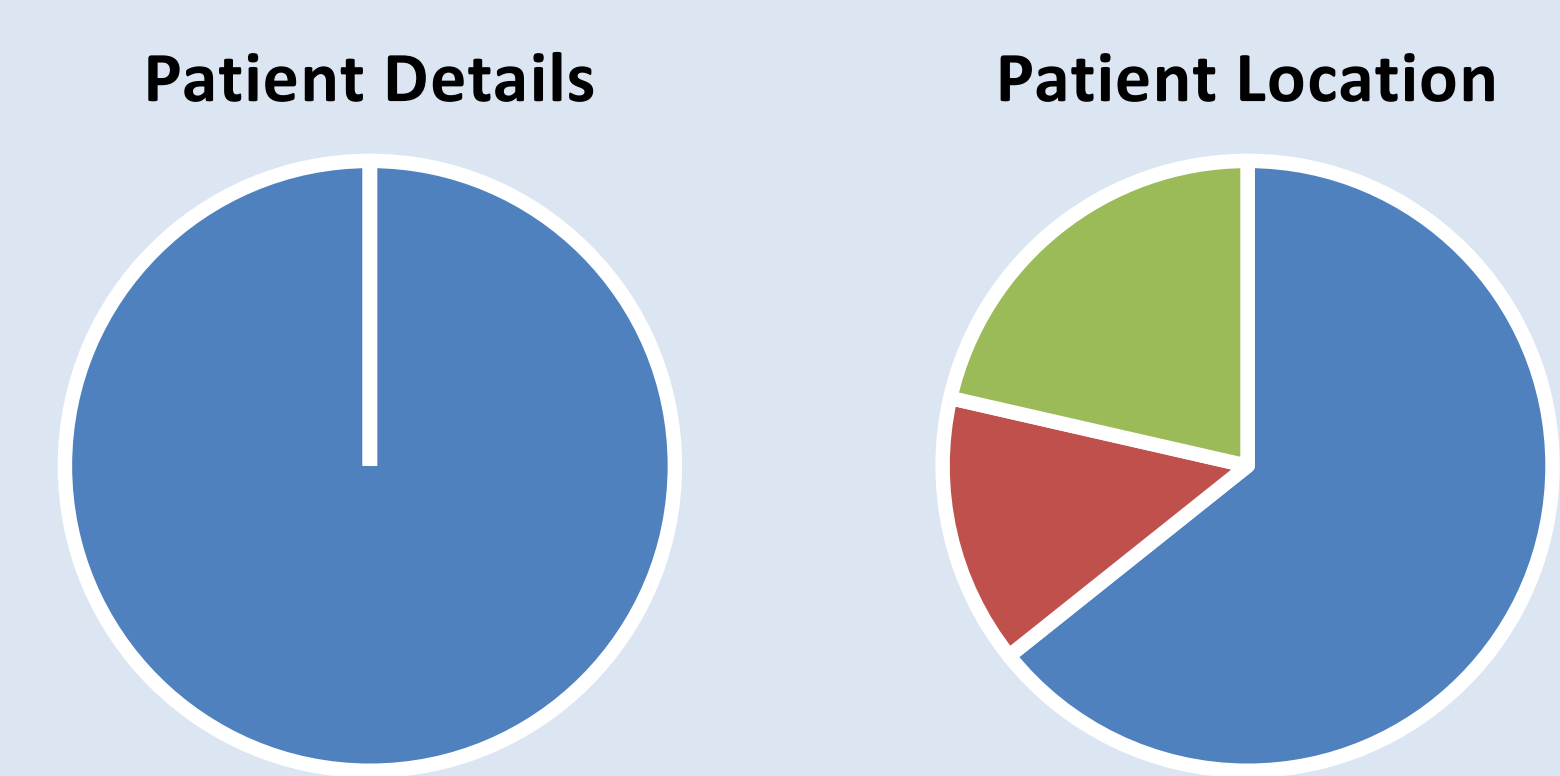

Estimated expected length of
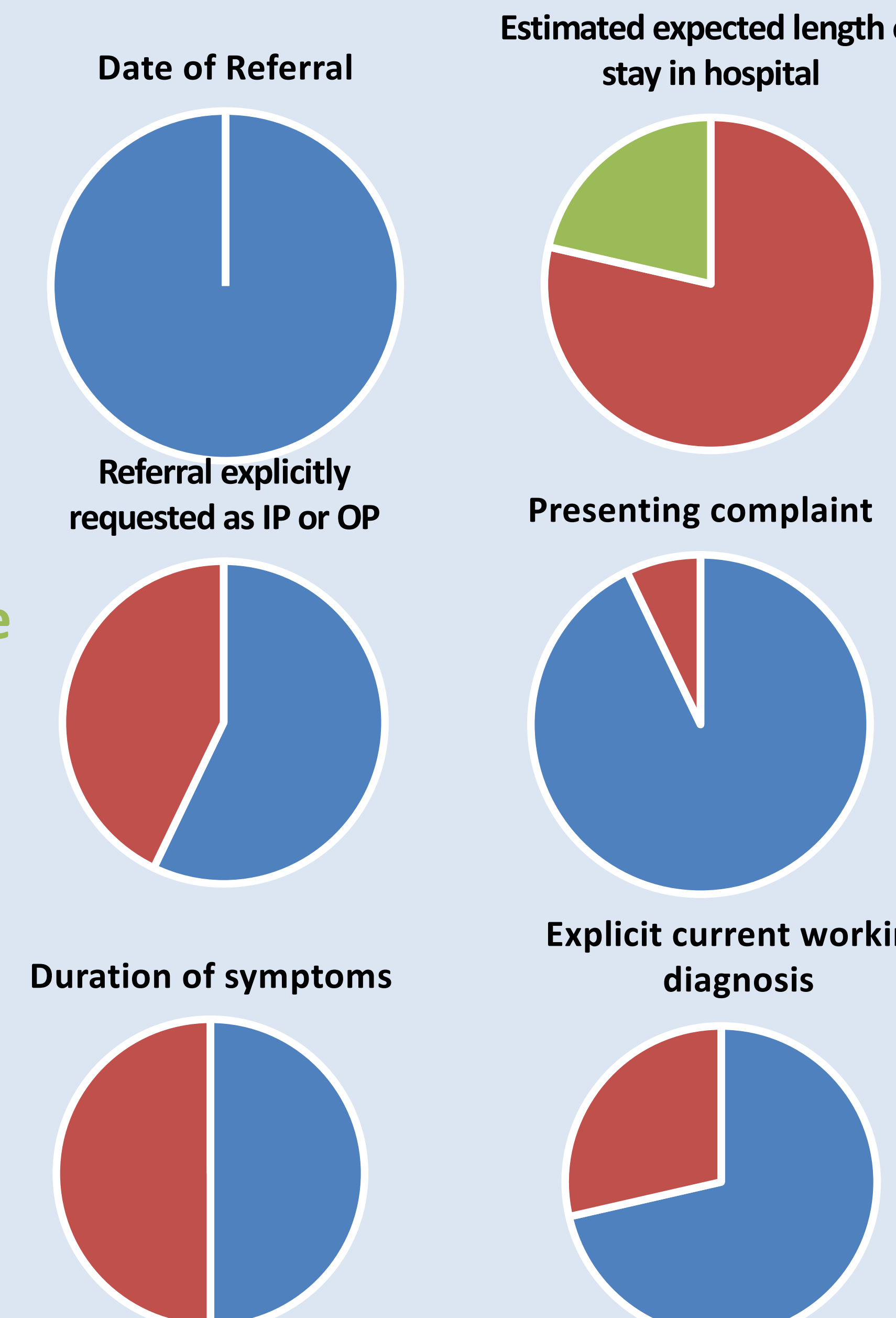

present

Not

applicable

Legend

Present

Not

Presenting complaint

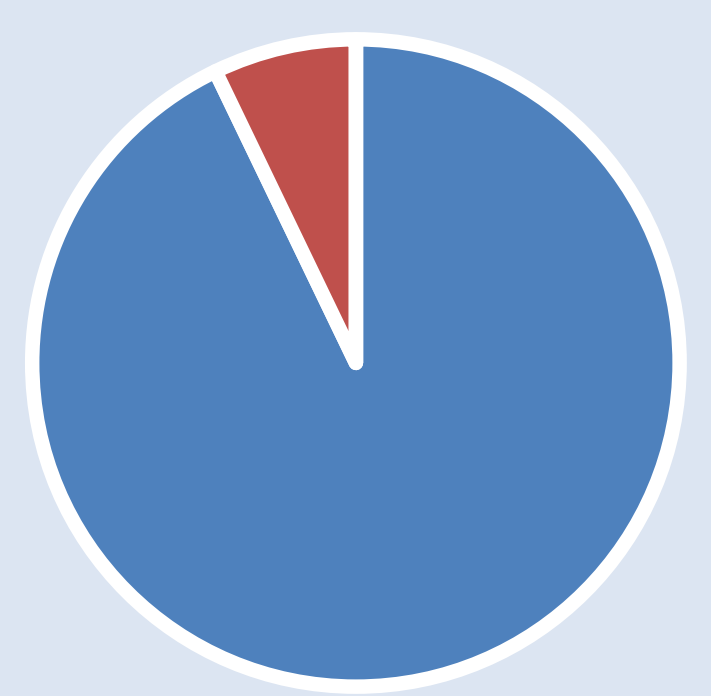

Explicit current working diagnosis

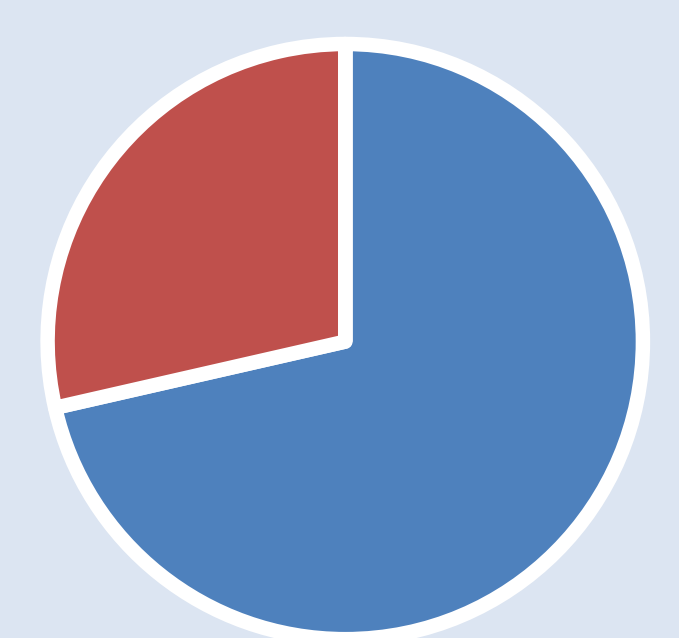

Figure 2. Charts depicting level of data included for paper referrals

\section{CONCLUSION}

- Due to the low compliance with the required minimum level of information, it is clear that the current system is not the most efficient or accurate for patients to be seen in the most appropriate manner.

- We suggest an online referrals system with set criteria that the referring clinician must complete prior to the referral being accepted.

- This system would allow for feedback to return to the referring clinician e.g. "will see as IP", "OPD requested", "referral rejected due to lack of information". 